\title{
Robust Segmentation of Medical Images Using Geometric Deformable Models and a Dynamic Speed Function
}

\author{
Benoit M. Dawant ${ }^{1}$, Shiyan $\operatorname{Pan}^{1}$, and Rui $\mathrm{Li}^{1}$ \\ ${ }^{1}$ Vanderbilt University, Department of Electrical Engineering and Computer Science, Box \\ 1662 Station B, Nashville, TN 37235 \\ \{Benoit.Dawant, Shiyan.Pan, Rui.Li\}@vanderbilt.edu
}

\begin{abstract}
A number of methods based on geometric deformable models have been proposed recently to segment medical images. These methods require the definition of a speed function that governs model deformation. In this paper we propose a new speed function that is modified dynamically as the front progresses. This new speed function is particularly well adapted to situations where edges are ill-defined, adjacent structures have comparable intensity values, and images are noisy. We illustrate qualitatively the performance of our approach on a variety of MR, CT, and ultrasound images. The examples show the generality of our approach and its insensitivity to parameters. We also evaluate it quantitatively on several CT scans of the liver.
\end{abstract}

\section{Introduction}

In recent years, geometric deformable models have been proposed as an alternative to parametric deformable models such as snakes originally proposed by Kass et al. [1]. In the geometric deformable model framework, the evolution of an initial contour toward the true object boundary is considered as a front propagation problem. This permits the use of the level set and fast marching methods introduced by Sethian [2] to model propagating fronts with curvature-dependent speeds. Following the level set formulation, the boundary of a 3D object is embedded as the zero-level set of a timedependent function $\Phi$. As time progresses, the value of the function $\Phi$ evolves and the boundary is given by its zero-level set. In practice, the function $\Phi$ is computed iteratively as follows:

$$
\Phi_{i, j, k}^{n+1}=\Phi_{i, j, k}^{n}-\Delta t \cdot F\left|\nabla_{i, j, k} \Phi_{i, j, k}^{n}\right|,
$$

in which $\mathrm{F}$ is the speed function, i.e., the function that specifies the speed at which the contour evolves along its normal direction. The main challenge when using front propagation methods is to produce an adequate speed function for a specific application. Malladi et al. [3] have proposed a general model for image segmentation defined as

$$
F=g_{I}\left(F_{0}-\varepsilon \kappa\right),
$$


in which $g_{I}$ is a multiplicative term derived from the image itself. It is used to stop the propagation of the contour near desired points such as points with high gradient or prespecified intensity values, $\kappa$ represents the curvature of the front and acts as a regularization term; $F_{0}$ is a constant; and $\varepsilon$ is a weighting parameter. But except if hard thresholds are set on the value of $g_{I}$ (i.e. if $g_{I}$ is set to zero when the gradient exceeds a certain value or when intensity values fall outside a predefined range), contours never stop completely at weak edges. Given enough time these will eventually "leak" outside the desired regions. This problem has been recognized and a solution has been proposed by Casselles et al. [4] who introduced an additional term in the speed function designed to attract contours toward boundaries. Although this term offers a partial solution to the leakage problem it is still not very well adapted to situations with weak edges in noisy and non-uniform images [5]. To address this problem speed functions that take into account probability density functions of regions inside and outside the structures of interest have been proposed $[5,6]$. This class of solution is well adapted to situations where adjacent structures have different intensity distributions but are challenged by applications that require the segmentation of structures surrounded by other structures with similar intensity values and separated by weak edges.

In this paper, we propose a new expression for the speed function that greatly increases the robustness of geometric deformable models for the segmentation of $2 \mathrm{D}$ and 3D noisy images with weak edges. The major difference between the approach we propose and previous approaches is the dynamic adaptation of the speed function. We call this new dynamic speed functions the accumulative speed function because its value depends on the path of the front. The next sections describe our approach and present qualitative and quantitative results we have obtained on a variety of medical images.

\section{Method}

\subsection{The Accumulative Speed Function}

In traditional speed functions, the terms related to the underlying image are static, i.e., they do change spatially but for a fixed location, they do not change as time progresses or as a function of the path followed by the front. To stop the front propagation at weak boundaries, speed functions have to be designed to decelerate the fronts very rapidly. This necessitates the choice of large values for the weights assigned to the gradient or intensity terms with the possible drawback of stopping the front at spurious edges. The novel approach we propose is to progressively slow the front down as it passes over the boundary points. In essence, we build memory into the process. If the front passes over one boundary point it slows down some. If it passes over two boundary points in sequence, it slows down more, etc., until it comes to a complete halt. This speed function allows the front to step over noisy points (for instance speckle noise in ultrasound images) while stopping at the object boundary even if it is not clearly defined. 


\subsection{Computation of the Accumulative Speed Function}

The algorithm we propose to implement our dynamic speed function is as follows:

1. Define a standard image-dependent static multiplicative term as described earlier and call it $g_{\text {bas }}$.

2. Define $g_{0}$ as the actual multiplicative term to be used in the speed function and let $g_{0}=g_{\text {bas }}$.

3. At each iteration, compute $\{(x, y, z) \mid \Phi(x, y, z)=0\}$, the zero level set of the embedding function. For every point in this set, retrieve $g_{b a s}(x, y, z)$ and extend it to a narrow band around the zero level set (the notion of extension is defined later). This creates an additional multiplicative term which we call $g_{\text {ext }}$

4. Let $g_{0}=g_{0} \cdot g_{\text {ext }}$

5. Use $g_{0}$ as the multiplicative term in the speed function that governs the propagation of the front. For example, to include curvature in the final speed function, define it as $F=g_{0}(1-\varepsilon \kappa)$

6. Compute $\Phi^{n+1}$, the value of the embedding function, using equation (1)

7. After a predefined number of iterations or if the zero level set does not move, stop. Otherwise, go to step 3

Step 3 in the algorithm requires the computation of an extended multiplicative term. Here, the notion of extension is the same as the one proposed by Adalsteinsson and Sethian [7] for velocities defined on zero level sets only. Loosely speaking, for voxels close to the zero level set, we set $g_{\text {ext }}(x, y, z)=g_{\text {bas }}(c(x, y, z))$ in which $c(x, y, z)$ is the closest point in the zero level set. Following Adalsteinsson's approach, this can be achieved by computing an extended multiplicative term that satisfies the following equation:

$$
\nabla g_{e x t} \cdot \nabla \varphi=0
$$

in which $\varphi$ is the standard signed distance function computed from the zero level set. This equation is solved using the Fast Marching Method as proposed by Sethian.

The key step in our algorithm is step 4. If a section of the front is over a region where the underlying static multiplicative term is small albeit non-zero, the actual multiplicative term decreases exponentially toward zero and the front stops within a few iterations. When this happens, the front is stopped permanently. Because of this, we do not need to design speed functions that are exactly zero to stop the front at the desired boundaries. We have the much easier task to design speed functions that have small values over the object boundaries. Examples will show that it lets us define generic speed functions that require tuning of very few parameters. 


\subsection{The Static Multiplicative Term $\mathrm{g}_{\text {bas }}$}

Our approach still requires the definition of an underlying static multiplicative term. The underlying multiplicative term we have used in all the experiments presented herein is simple and it is shown in Figure 1 (the left panel of the figure shows its analytical expression; the function is plotted on the right panel). In this equation, $I_{\min }$ and $\mathrm{I}_{\max }$ are two parameters used to specify the minimum and maximum intensity values expected in the structure of interest, $\sigma$ is the standard deviation of these intensity values, and $\gamma$ is used to control the rate at which $g$ decreases; the smaller the value of $\gamma$, the faster the reduction in speed at weak boundaries.

$$
g_{\text {bas }}= \begin{cases}\exp \left[\frac{-\left(I-I_{\min }\right)^{2}}{\gamma \sigma_{I}^{2}}\right] & I<I_{\min } \\ 1 & I_{\min }<I<I_{\max } \\ \exp \left[\frac{-\left(I-I_{\max }\right)^{2}}{\gamma \sigma_{I}^{2}}\right] & I>I_{\max }\end{cases}
$$

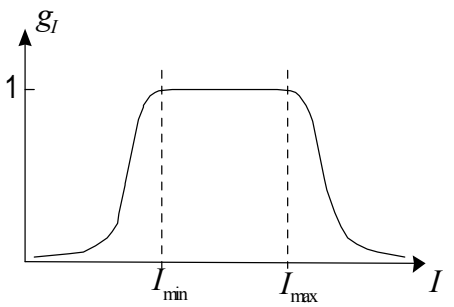

Fig. 1. Function used to define the static multiplicative term

\section{Results}

\subsection{Qualitative Results}

We have experimented with this multiplicative term combined with the new dynamic update scheme we have developed on a series of segmentation tasks ranging from the segmentation of structures and substructures in MR images of the brain, the ventricles in MR and ultrasound images of the heart, to the liver in MR images of mice. In every case the same underlying multiplicative term was used and the only parameters that were adjusted were $I_{\min }, I_{\max }$, and $\varepsilon$, the parameter used to weigh the contribution of the curvature term; $\gamma$ and $\sigma$ were kept constant. The overall speed function we have used for these experiments is as in (2) with $F_{0}=1$. Note that the formulation we propose does not include any term related to the intensity gradient which has little meaning in noisy images or in images with weak edges. 

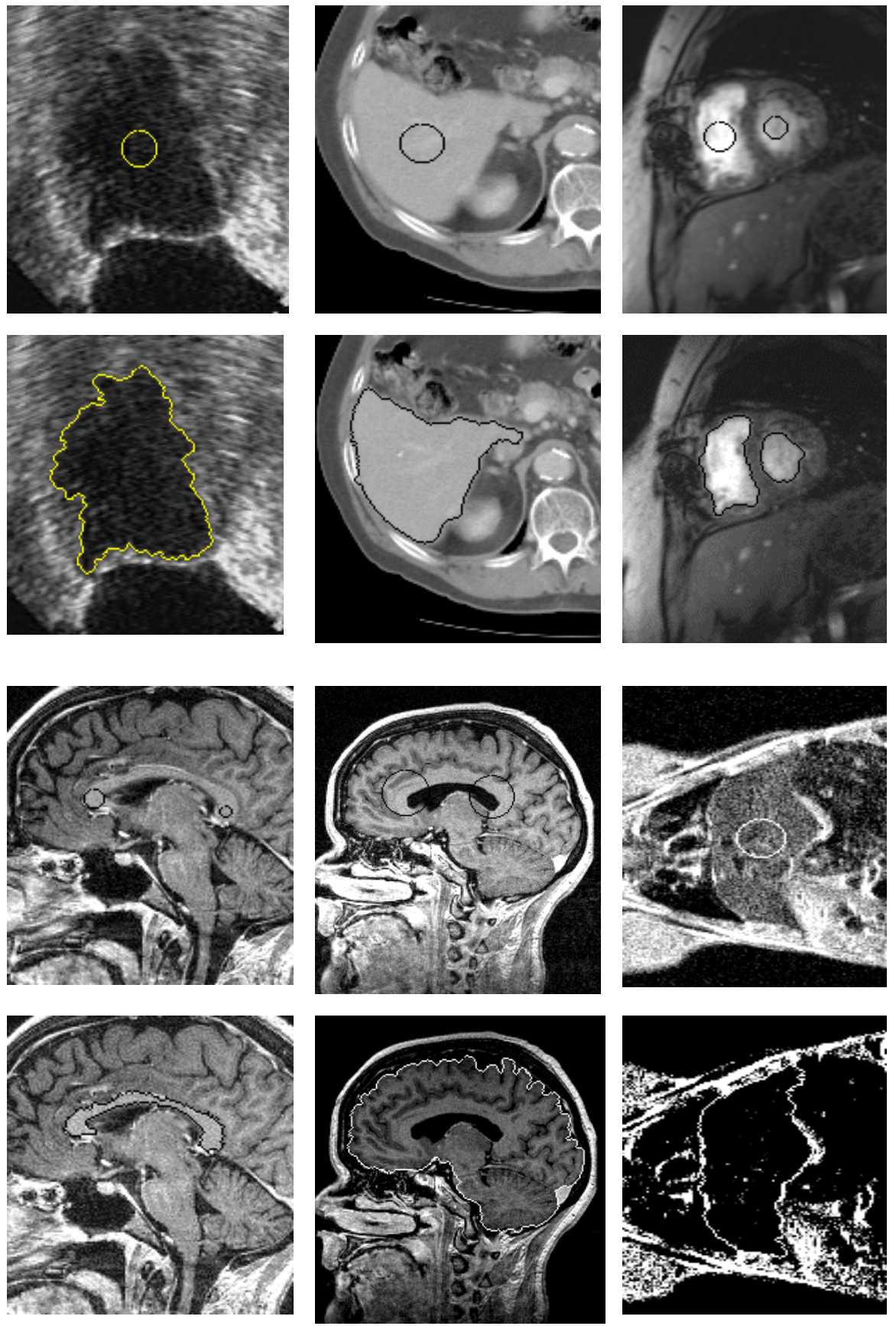

Fig. 2. Illustrative examples of results obtained with our method. For each pair of images, the top one shows the original contour; the bottom one shows the final contour. 


\begin{tabular}{|l|l|l|l|l|}
\hline & Imin & Imax & Epsilon & Gamma \\
\hline Heart US & 10 & 60 & 3.0 & 100 \\
\hline Liver CT & 90 & 200 & 3.0 & 100 \\
\hline Heart MR & 140 & 400 & 3.0 & 100 \\
\hline Corp. Call. & 70 & 85 & 2.0 & 100 \\
\hline Brain MR & 40 & 85 & 3.0 & 100 \\
\hline Mouse MR & 25 & 50 & 3.0 & 100 \\
\hline
\end{tabular}

Table 1. Parameters used to generate the resutls shown in Figure 2.

A number of movies showing results we have obtained can be seen at http://www.vuse.vanderbilt.edu/ dawant/levelset_examples. In each of these movies, the contours have been initialized with small circles shown on the first frames of the movies and the end of the movies shows the final contours. Figure 2 presents a static view of some of these results obtained on a variety of images. From left to right and top to bottom, this figure shows results obtained on an ultrasound image of the heart, a CT image of the liver, an MR image of the heart, an MR image of the head focused on the corpus callosum, an MR image of the head showing the entire brain, and an MR image of a mouse focused on the liver. In every instance, a pair of image is presented. The top one shows the original contour, the bottom one shows the final contour. For the final mouse image, the contrast and intensity values have been adjusted to highlight the final contour because the amount of noise present in the image prevents the simultaneous display of the tissues and the contour on a gray level image. Table 1 lists the parameters that were used in each case. This figure clearly shows that the results are largely insensitive to the choice of parameters. The value of $\varepsilon$ had to be reduced for the corpus callosum image because the structure is narrow and a larger value for the curvature term did prevent the propagation of the front. Further experiments have also revealed that the results are insensitive to the precise values of $I_{\text {min }}$ and $\mathrm{I}_{\max }$. The human liver and corpus callosum images illustrate the performance of our algorithm for the segmentation of structures with similar intensity values that are separated by weak edges. In the liver image, the liver and the kidney have similar intensity values and are separated by a blurred edge. Observe how the front stopped at the correct position and also that it did not penetrate the vena cava. The corpus callosum image shows the posterior part of this structure as a region of white matter surrounded by a thin layer of gray matter and then white matter again. Here, the contour stopped correctly at the narrow white/gray matter interface. The mouse image has been chosen to show the behavior of the algorithm on images with very poor signalto-noise ratio.

\subsection{Quantitative Results}

To validate the proposed method quantitatively, we tested it on five abdominal CT scans, three of which are from normal subjects and two others contain abnormal livers with tumors for a total of 280 slices. Scans were selected to include livers with differ- 
ent size, shape, and position in the abdomen. Experiments were conducted in $2 \mathrm{D}$ and $3 \mathrm{D}$ in all five scans. The voxel size for all scans is $0.625 \mathrm{~mm} \times 0.625 \mathrm{~mm} \times 3 \mathrm{~mm}$. In $2 \mathrm{D}$, the algorithm was initialized by drawing a small circle within the liver in each slice. In slices where the liver consists of several disconnected structure, one small circle was placed within each structure. The $3 \mathrm{D}$ algorithm was initialized by placing a sphere somewhere inside the liver. For each of these volumes, we kept the same parameters except for $I_{\min }$ and $I_{\max }$ which were adjusted to compensate for slight contrast differences between volumes due to the injection of a contrast agent.

Even though the speed function and update scheme we propose are able to stop contours when edges are ill defined, we found it to be insufficient for this particular application. Indeed, between the ribs, the edge at the liver/muscle is virtually non-existent and both tissues are iso-intense. The approach we have taken to address this problem is to constrain the propagation using a priori anatomic information. We locate the skin/air interface and the position of the ribs. We compute an average distance between the ribs and the skin and we constrain the propagation of the front using this distance.

All segmentation results were compared with manual delineations inspected and approved by an experienced surgeon. For each point in the plots shown in Figure 3 , the $\mathrm{X}$ and $\mathrm{Y}$ coordinates correspond to the area of manual and automatic segmentations in the same slice, respectively. The majority of points cluster near the straight line with slope of 1 and passing through the origin, which indicates a good match between the

two sets of segmentations. Some scattered points are found above the line in the $3 \mathrm{D}$ plot and below the line in both the $3 \mathrm{D}$ and 2D plots. Points above the line repre-
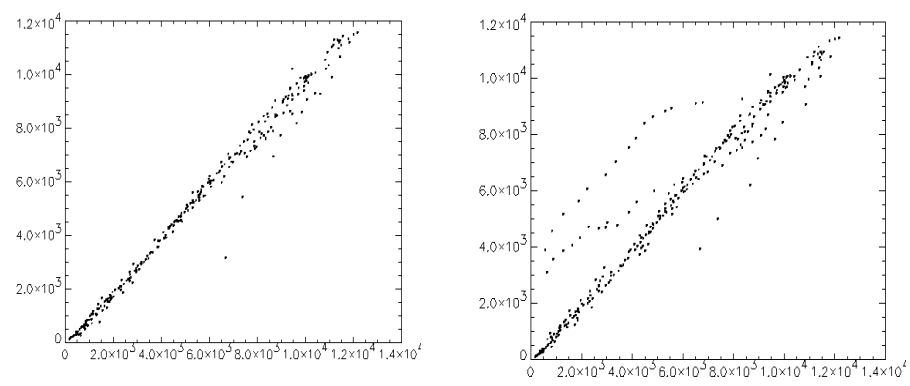

Fig. 3. Areas of automatic contours versus areas or manual contours for every slice; left 2D, right 3D.

sent slices in which leakage did occur. Points below the line represent slices in which the automatic segmentation was unable to capture the entire liver.

This figure shows that the results we have obtained with both the $2 \mathrm{D}$ and $3 \mathrm{D}$ versions of our algorithm are in close agreement with manual delineation, it also indicates a rate of failure that is slightly higher for the $3 \mathrm{D}$ version than for the $2 \mathrm{D}$ version. Closer investigation of this phenomenon has shown that this is due to one image volume in which leakage occurred across slices. It is caused by the anisotropic nature of the image volumes we have used (the slice thickness of the images is 5 times larger than the in-plane resolution). In this volume, the boundary between the heart and the liver is not resolved between two slices. The $3 \mathrm{D}$ algorithm leaked from the liver into the heart between these two slices and then expanded into the heart in 3D. This, in turn, caused large segmentation errors on a number of slices. For the other volumes, $2 \mathrm{D}$ and $3 \mathrm{D}$ results are identical. Movies that illustrate the behavior of the algorithm 
we propose for liver segmentation can be viewed at the aforementioned URL (files liver2d.mpg and liver3d.mpg).

\section{Discussion}

The dynamic update scheme we propose for image segmentation using geometric deformable models permits the $2 \mathrm{D}$ and $3 \mathrm{D}$ segmentation of vastly different images and structures with only few parameter adjustments. Examples presented show that the algorithm we propose is able to cope with ultrasound images that are notoriously difficult to segment because of speckle noise as well as with images with low signal-tonoise ratio and poorly defined edges. The key feature of our algorithm is its ability to stop the propagation of the front even if the underlying multiplicative term in nonzero. If the front encounters one voxel with a low value for the multiplicative term, it slows down but does not stop. But if it passes several voxels in a row all with a low value it slows down exponentially until it comes to a complete halt. This property led us to call the speed function we propose the accumulative speed function because the likelihood of being at a real boundary point increases if several possible boundary points are encountered in sequence.

\section{References}

1. M. Kass, A. Witkin, and D. Terzopoulos, "Snakes: active contour models." Int'l J. Comp. Vis., 1(4):321-331, 1987

2. J.A. Sethian, "Numerical algorithms for propagating interfaces: Hamilton-Jacobi equations and conservation laws", J. of Differential Geometry, vol. 31, pp. 131-161, 1990

3. R. Malladi, J.A. Sethian, and B.C. Vemuri, "Shape modeling with front propagation: A level set approach", IEEE Trans. PAMI, 17(2):158-175, 1995

4. V. Caselles, R. Kimmel, and G. Sapiro, "Geodesic active contours", Proc. Int'l Conf. Comp. Vis., pp. 694-699, 1995

5. C. Xu, A. Yezzi Jr., and J. Prince, "On the relationship between parametric and geometric active contours", Technical Report JHU/ECE 99-14, Dec. 1999

6. C. Baillard and C. Barillot, "Robust 3D segmentation of anatomical structures with level sets", Proceedings of MICCAI 2000, pp. 237-245, 2000.

7. D. Adalsteinsson and J.A. Sethian, "The fast construction of extension velocities in level set methods", L. Comp. Phys., 138, 1, pp. 193-223, 1997. 